\title{
Optimized Multiple Description Lattice Vector Quantization for Wavelet Image Coding
}

\author{
Huihui Bai, Ce Zhu, Senior Member, IEEE, and Yao Zhao, Member, IEEE
}

\begin{abstract}
Multiple description (MD) coding is a promising alternative for robust transmission of information over non-prioritized and unpredictable networks. In this paper, an effective MD image coding scheme is introduced based on the MD lattice vector quantization (MDLVQ) for the wavelet transformed images. In view of the characteristics of wavelet coefficients in different frequency subbands, MDLVQ is applied in an optimized way, including an appropriate construction of wavelet coefficient vectors, the optimization of MDLVQ encoding parameters such as the choice of sublattice index values and the quantization accuracy for different subbands. More importantly, optimized side decoding is employed to predict lost information based on inter-vector correlation and an alternative transmission way for further reducing side distortion. Experimental results validate the effectiveness of the proposed scheme with better performance than some other tested MD image codecs including that based on optimized MD scalar quantization.
\end{abstract}

Index Terms-Image coding, lattice vector quantization, multiple description (MD) coding, wavelet transform.

\section{INTRODUCTION}

$\mathbf{N}$ ETWORK congestion and delay sensibility pose great challenges for multimedia communication system design. This creates a need for coding approaches combining high compression efficiency and robustness. Multiple description (MD) coding has emerged as an attractive framework for robust transmission over unreliable channels. It can effectively combat packet loss without any retransmission thus satisfying the demand of real time services and relieving the network congestion [1]. Multiple description coding encodes the source message into several bit streams (descriptions) carrying different information which can then be transmitted over separate channels. If only one channel works, the only description can be individually decoded to guarantee a minimum fidelity in the reconstruction at the receiver. When more channels work, the descriptions from these channels can be combined to yield a higher fidelity reconstruction.

The MD versions of transforms [2] and quantizers [3]-[8] are the two main techniques for the design of MD image coding. An MD image coder using pairwise correlation transform is presented in [2]. A design of MD scalar quantizers (MDSQ)

\footnotetext{
Manuscript received November 14, 2005; revised August 28, 2006. This work was supported in part by National Natural Science Foundation of China (No. 90604032, No. 60373028), Specialized Research Fund for the Doctoral Program of Higher Education, Program for New Century Excellent Talents in University, Specialized Research Foundation of BJTU, 973 program (No.2006CB303104). This paper was recommended by Associate Editor S.-U. Lee.

H. Bai and Y. Zhao are with Institute of Information Science, Beijing Jiaotong University, Beijing 100044, China (e-mail: luckybhh@126.com, yzhao@center. njtu.edu.cn).

C. Zhu is with School of Electrical and Electronic Engineering, Nanyang Technological University, 639798 Singapore (e-mail: eczhu@ntu.edu.sg).

Color versions of one or more of the figures in this paper are available online at http://ieeexplore.ieee.org.

Digital Object Identifier 10.1109/TCSVT.2007.898646
}

for image coding is described in [3], which exploits a suboptimal algorithm to allocate rate subject to global constraints on the coding bit rate and side distortion. In [5], an MD image coding algorithm is developed also based on MDSQ with optimization of MDSQ parameters, which produces similar peak signal-to-noise (PSNR) values with about $50 \%-60 \%$ of the bit rates required by the two above mentioned MD image coders. In [6], a modified MDSQ (MMDSQ) is designed in two stages, that is, the first stage is a basic MDSQ stage while the second stage is a finer central quantizer to quantize the residuals from the first one. The MMDSQ is applied to the Tarp filter image coder with classification for embedding (TCE) [10], which is reported to have achieved better performance. In addition, to be compatible with the image coding standards, some other methods like that in [9] have designed MD image coders without using any special transforms or MD quantizers. In contrast, the MD coding scheme in [9] directly utilizes streams produced by a JPEG2000 encoder and exploits the rate-allocation of JPEG2000 streams to produce MD streams.

We attempt to apply MD lattice vector quantization (MDLVQ) for the design of a more effective two-channel MD (or two-description) image coder. We developed a preliminary MDLVQ based image coder in [8]. In this paper more effective enhancements are considered to substantially improve the performance of MDLVQ based image coding. Several factors which are important and of significant impact to the performance are taken into consideration for optimized MDLVQ encoding and decoding. Firstly, the forming of coefficient vectors needs to adapt to different correlating characteristics between wavelet coefficients in different subbands. Secondly, like the optimization for the MD scalar quantization (MDSQ) [5], the MDLVQ encoding parameters need to be optimized in terms of rate-distortion performance in view of the varying importance of different subbands. More importantly, the reconstruction quality from the conventional MDLVQ side decoders can be significantly improved by utilizing inter-vector correlation and predicting the lost information when necessary for further reducing the side distortion. To realize the optimized side decoding, an alternative transmission of MDLVQ labels is developed.

The rest of this paper is organized as follows. In Section II, an overview of the proposed MD coding scheme is given. In Section III, the optimization of MDLVQ encoding and decoding is presented in detail. The performance of the proposed scheme is examined against the other coders in Section IV. We conclude the paper in Section V.

\section{OVERVIEW OF PROPOSED MD SCHEME}

Fig. 1 illustrates our scheme and a step-by-step recipe is explained as follows. Here, we consider two balance channels, that is, the bit rate of two descriptions and the side distortions for the two side decoders are approximately the same. 


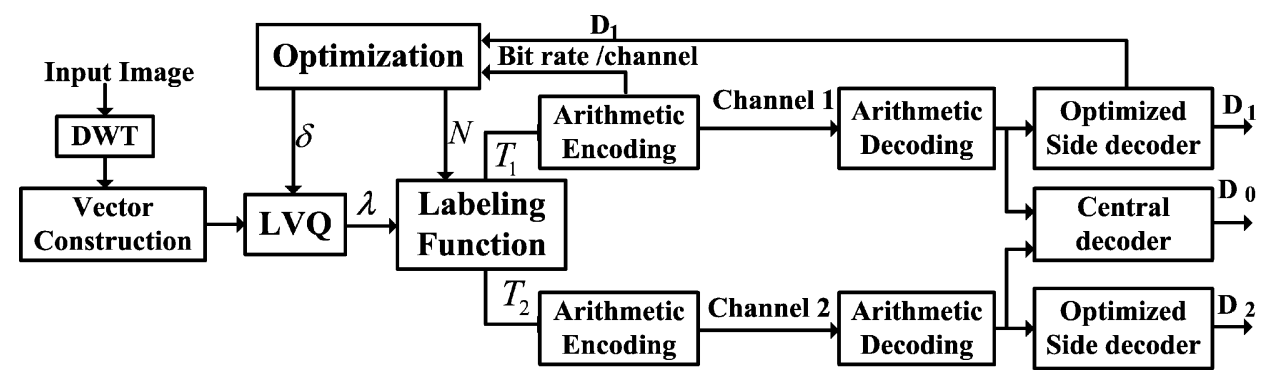

Fig. 1. Block diagram of our proposed scheme.

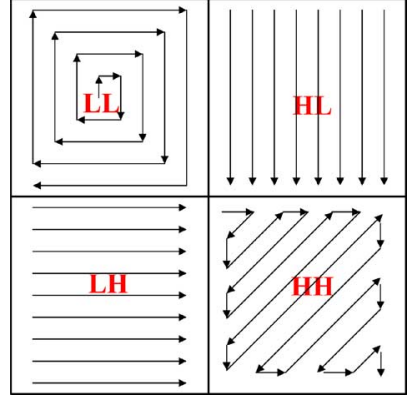

Fig. 2. Vector construction in different subbands.

\section{Step 1) Wavelet Decomposition}

A given input image is decomposed into subbands (subband 1 , subband $2, \ldots$, subband $m$, denoted by $s_{i}, i=$ $1,2, \ldots, m)$, by applying a wavelet transform. Following that done in [5], small wavelet coefficients are set to zeros by applying an appropriate threshold.

\section{Step 2) Vector Construction}

After wavelet decomposition, correlation still remains between coefficients in the subbands. Grouping the coefficients appropriately can exploit intra-vector redundancy well. During wavelet decomposition, subbands arise from separable application of vertical and horizontal filters, denoted as LL, HL, $\mathrm{LH}, \mathrm{HH}$, respectively [11]. It is known that wavelet coefficients in different subbands have different directional correlation. For example, vertical correlation exhibits in HL coefficients, horizontal correlation in $\mathrm{LH}$, diagonal correlation in $\mathrm{HH}$ and neighboring correlation of low-frequency components in LL. Therefore, it is more efficient to group coefficients in different subbands according to their directional correlation. Fig. 2 shows our scheme for vector construction: HL is scanned to form vectors along vertical direction, LH is scanned in horizontal direction and $\mathrm{HH}$ is scanned in zigzag way. In addition, spiral scan is also applied in LL subband considering the strong correlation among neighboring coefficients. Predictive vector quantization can be applied to further exploit these correlations.

\section{Step 3) Lattice Vector Quantizer (LVQ)}

In this paper, lattice $A_{2}$ is used for lattice vector quantization (LVQ). It has been shown [4] that in the two-channel case, MDLVQ using $A_{2}$ exhibits better performance than that based on $Z_{2}$ in terms of central and side distortions. $A_{2}$ is equivalent or similar to the hexagonal lattice [12]. The hexagonal lattice can be spanned by the vectors $(1,0)$ and $(-1 / 2, \sqrt{3} / 2)$, and the generator matrix is

$$
G=\left[\begin{array}{cc}
1 & 0 \\
\frac{-1}{2} & \frac{\sqrt{3}}{2}
\end{array}\right]
$$

Every pair of coefficients in each subband is formed as a 2-D vector according to the grouping way in Step 2. A lattice vector quantizer with a quantization "volume-size" (like the step-size in scalar quantization) is applied to such 2-D vectors, thus producing a quantized symbol $\lambda, \lambda \subset A_{2}$. It is known that the VQ encoding complexity increases with dimensionality and codebook size. Here we use the lowest dimension vector, i.e., 2-D vector. Moreover, LVQ encoding can be implemented by a fast quantizing algorithm [12] which does not require performing the computation-intensive nearest neighbor search based on squared distance calculation. In the fast encoding algorithm [12], only two matrix multiplications are required for vector mapping between a $2-\mathrm{D}$ vector and a 3-D vector, and a modification may be needed for the mapped 3-D vector to make the sum of its 3-D values zero. In this way, the complexity of LVQ on $A_{2}$ is considered very low. In addition, another fast quantizing algorithm in [12] may be a better choice to accelerate LVQ encoding further. In view that the hexagonal lattice is the union of two rectangular lattices, the encoding can be simply achieved by finding the nearest point in each rectangular sublattice and selecting the nearer of these two points.

\section{Step 4) Labeling Function With Alternative Transmission}

Information about a quantized point $\lambda$ is mapped to two representations and then sent across two channels, subjected to bit rate constraints imposed by each individual channel. This is done by a labeling function [7] followed by arithmetic encoding. The labeling function maps $\lambda \subset \Lambda$ to a pair $\left(\lambda_{1}^{\prime}, \lambda_{2}^{\prime}\right) \in \Lambda^{\prime} \times \Lambda^{\prime}$, where $\Lambda^{\prime}$ is a sublattice of $\Lambda$ with the index $N, N=\left|\Lambda / \Lambda^{\prime}\right|$. The index $N$ determines the coarse degree of the sublattice which can control the amount of redundancy in the MD coder [4]. In Section III, optimization for the index $N$ and the LVQ quantization "volume-size" (in Step 3) will be presented in detail.

Fig. 3 is an example of a $A_{2}$ sublattice with index $N=13$. In the case of $N=13$, we can obtain a labeling function as in Table I, where each fine lattice point $\lambda$ is mapped to a unique label $\left(\lambda_{1}^{\prime}, \lambda_{2}^{\prime}\right)$, with $\lambda_{1}^{\prime}$ and $\lambda_{2}^{\prime}$ being two sublattice points as close to $\lambda$ as possible. Note that the proposed mapping scheme shown in the table is slightly different from the index assignment developed by Servetto, Vaishampayan and Sloane [4] (known as SVS technique). In our proposed scheme, $\lambda_{1}^{\prime}$ is always closer 


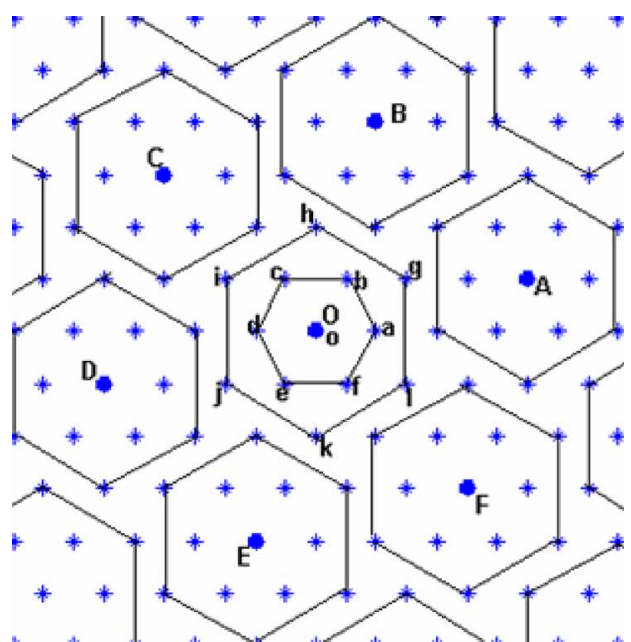

Fig. 3. Example of sublattice with index 13:fine lattice points are labeled by $a, b, c, \ldots, l$, and sublattice points are $\mathrm{A}, \mathrm{B}, \mathrm{C}, \ldots, \mathrm{F}$.

TABLE I

LABELING FUNCTION WITH $N=13$

\begin{tabular}{cccc}
\hline \hline Lattice Point $\lambda$ & Label & Lattice Point & Label \\
\hline $\mathrm{o}$ & $(\mathrm{O}, \mathrm{O})$ & & \\
$\mathrm{a}$ & $(\mathrm{O}, \mathrm{A})$ & $\mathrm{d}$ & $(\mathrm{O}, \mathrm{D})$ \\
$\mathrm{b}$ & $(\mathrm{O}, \mathrm{B})$ & $\mathrm{e}$ & $(\mathrm{O}, \mathrm{E})$ \\
$\mathrm{f}$ & $(\mathrm{O}, \mathrm{F})$ & $\mathrm{c}$ & $(\mathrm{O}, \mathrm{C})$ \\
$\mathrm{g}$ & $(\mathrm{B}, \mathrm{F})$ & $\mathrm{j}$ & $(\mathrm{E}, \mathrm{C})$ \\
$\mathrm{h}$ & $(\mathrm{C}, \mathrm{A})$ & $\mathrm{k}$ & $(\mathrm{F}, \mathrm{D})$ \\
$\mathrm{i}$ & $(\mathrm{D}, \mathrm{B})$ & 1 & $(\mathrm{~A}, \mathrm{E})$ \\
\hline \hline
\end{tabular}

to $\lambda$, thus $\lambda_{1}^{\prime}$ is denoted as the near sublattice point and $\lambda_{2}^{\prime}$ the far sublattice point. To strike a balance of reconstruction quality with any single description sequence, $\lambda_{1}^{\prime}$ and $\lambda_{2}^{\prime}$ are alternately transmitted over two channels.

As a simple example, if we have a quantized sequence of fine lattice points $\{\lambda(1), \lambda(2), \ldots, \lambda(8)\}=$ $\{\mathrm{a}, \mathrm{a}, \mathrm{a}, \mathrm{b}, \mathrm{b}, \mathrm{b}, \mathrm{i}, \mathrm{i}\}$, then the two sequences of sublattice points using the labeling function in Table $\mathrm{I}$ are $\left\{\lambda_{1}^{\prime}(1), \lambda_{1}^{\prime}(2), \ldots, \lambda_{1}^{\prime}(8)\right\}=\{\mathrm{O}, \mathrm{O}, \mathrm{O}, \mathrm{O}, \mathrm{O}, \mathrm{O}, \mathrm{D}, \mathrm{D}\}$ and $\left\{\lambda_{2}^{\prime}(1), \lambda_{2}^{\prime}(2), \ldots, \lambda_{2}^{\prime}(8)\right\}=\{\mathrm{A}, \mathrm{A}, \mathrm{A}, \mathrm{B}, \mathrm{B}, \mathrm{B}, \mathrm{B}, \mathrm{B}\}$. Based on the alternative transmission scheme, the sequence

$$
\begin{aligned}
\left\{T_{1}\right\} & =\left\{\lambda_{1}^{\prime}(1), \lambda_{2}^{\prime}(2), \lambda_{1}^{\prime}(3), \lambda_{2}^{\prime}(4), \ldots, \lambda_{1}^{\prime}(7), \lambda_{2}^{\prime}(8)\right\} \\
& =\{\mathrm{O}, \mathrm{A}, \mathrm{O}, \mathrm{B}, \mathrm{O}, \mathrm{B}, \mathrm{D}, \mathrm{B}\}
\end{aligned}
$$

is transmitted over channel 1 and

$$
\begin{aligned}
\left\{T_{2}\right\} & =\left\{\lambda_{1}^{\prime}(1), \lambda_{2}^{\prime}(2), \lambda_{1}^{\prime}(3), \lambda_{2}^{\prime}(4), \ldots, \lambda_{1}^{\prime}(7), \lambda_{2}^{\prime}(8)\right\} \\
& =\{\mathrm{A}, \mathrm{O}, \mathrm{A}, \mathrm{O}, \mathrm{B}, \mathrm{O}, \mathrm{B}, \mathrm{D}\}
\end{aligned}
$$

over channel 2. The alternative transmission way is exploited in predicting lost description in the optimized side decoding, which will be explained in Section III-B.

A general and widely used context-based arithmetic codec [13] is applied to code the sequences of $\left\{T_{1}\right\}$ and $\left\{T_{2}\right\}$ before transmission. The arithmetic coding used in our scheme is based on a three-order "finite-context" model, that is, three previous symbols make up the context.

\section{Step 5) Central Decoder and Side Decoder}

At the receiver, if both descriptions are received, the two descriptions can be processed by the central decoder after arithmetic decoding and the sequence of fine lattice points $\{\lambda\}$ can be reconstructed with the central distortion. However, if either of descriptions is lost, the conventional side decoder can only produce $\lambda_{1}^{\prime}$ or $\lambda_{2}^{\prime}$ as an approximate to $\lambda$, leading to a larger side distortion. In contrast, we can obtain a better side decoding result by performing lost information prediction when necessary, based on the neighboring inter-vector correlation of wavelet coefficients and the above mentioned alternative transmission scheme. The design of the optimized side decoder with prediction will be elaborated in the subSection III-B.

\section{MDLVQ ENCODING AND DECODING OPTIMIZATION}

\section{A. Encoding Parameter Optimization}

In MDLVQ image encoding, there are two important factors which will affect the reconstruction image quality and the bit rate. The first one is the area of hexagonal lattice (in Step 3), i.e., the quantization "volume-size" used in LVQ, while the other is the choice of sublattice index (in Step 4).

Since the lattice $A_{2}$ is the space which can be spanned by two vectors $(1,0)$ and $(-1 / 2, \sqrt{3} / 2)$, the area of the hexagonal lattice is determined by the two vectors. However, we can keep the shape of the hexagonal lattice and change its area by multiplying the generator matrix $G$ by a factor $\delta,(\delta \in R, \delta>0)$. The parameter $\delta$ in the LVQ is similar to the step-size in scalar quantization (SQ). By changing $\delta$, the central distortion $D_{0}$ and its associated bit rate can be adjusted.

For the lattice $A_{2}$, the choice of index $N$ will not change the central distortion $D_{0}$ for a given $\delta$. However, the side distortion $D_{1}$ and $D_{2}$ will be sensitive to the value of $N$. When the index $N$ increases, $D_{0}$ has no change but $D_{1}$ and $D_{2}$ will increase significantly. On the other hand, the bit rates associated with $D_{1}$ and $D_{2}$ will decrease with the increase of $N$. The index $N$ is analogous to the number of diagonal of index assignment in MDSQ [5]. In MDSQ, the increasing of the number of diagonal will have severe impact on $D_{1}$ and $D_{2}$ and their associated rates while $D_{0}$ does not change. It is desired to find the optimal parameters $\delta$ and $N$ for striking the best trade-off among central distortion, side distortion and their associated bit rates. With the analysis of analogies between MDLVQ and MDSQ, we can perform the optimization of parameters $\delta$ and $N$ in MDLVQ encoding like the optimization way for MDSQ encoding in [5]. Therefore, we can formulate the MD design problem as yielding optimal performance in the presence of the constraints of the side distortion and its bit rate. To facilitate the description, some notations are defined in the following.

Let $I$ denotes an image, and $S=\left\{s_{1}, s_{2}, \ldots, s_{m}\right\}$ its $m$ wavelet subbands after the decomposition. $\delta_{S}$ refers to the magnified degree of the lattice area (i.e., quantization "volume-size") used for all the subbands. $N_{S}=$ $\left\{N_{s_{i}} \mid i=1,2, \ldots, m\right\}$ represents the set of the index numbers used in the labeling function for different subbands. $D_{0}\left(S, \delta_{S}, N_{S}\right), D_{1}\left(S, \delta_{S}, N_{S}\right)$ and $D_{2}\left(S, \delta_{S}, N_{S}\right)$ denote the mean squared errors (MSE) from the central decoder and the side decoders for the input image $I$, respectively, given the 


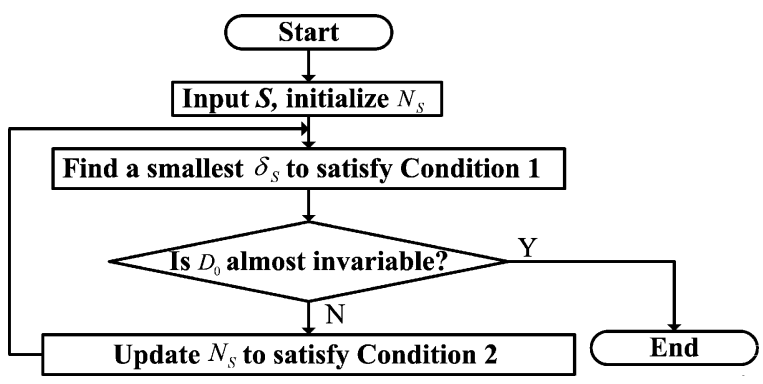

Fig. 4. Encoding optimization process.

lattice vector quantizers with parameter $\delta_{S}$ and the index set $N_{S} \cdot R_{1}\left(S, \delta_{S}, N_{S}\right)$ and $R_{2}\left(S, \delta_{S}, N_{S}\right)$ are the bit rates for encoding each description of $I$, respectively.

Our goal is to find the optimal parameters $\delta_{S}$ and $N_{S}$ in solving the following optimization problem:

$$
\begin{array}{r}
\min _{\delta_{S}, N_{S}} D_{0}\left(S, \delta_{S}, N_{S}\right) \\
\text { subject to }
\end{array}
$$

Condition $1: R_{1}\left(S, \delta_{S}, N_{S}\right)=R_{2}\left(S, \delta_{S}, N_{S}\right) \leq R_{\text {budget }}$

Condition $2: D_{1}\left(S, \delta_{S}, N_{S}\right)=D_{2}\left(S, \delta_{S}, N_{S}\right) \leq D_{\text {budget }}$

where $R_{\text {budget }}$ is the available bit rate to encode each description and $D_{\text {budget }}$ is the maximum distortion acceptable for single-channel reconstruction. The encoding optimization module in Fig. 1 is based on the above functions. With the constraints on the bit rate per channel and the side distortion, $\delta_{S}$ and $N_{S}$ are adjusted accordingly to minimize the central distortion.

The optimization for the problem is carried out in an iterative way. The basic algorithm shown in Fig. 4 is to make use of the monotonicity of both $R$ and $D$ as the functions of $\delta_{S}$. Firstly, after initialization a smallest $\delta_{S}$ is searched to minimize $D_{0}$ subject to Condition 1 . Secondly, according to Condition 2, we can update $N_{S}$ sequentially from high frequency subbands to low ones. Then the updated $N_{S}$ affects $R_{1}\left(S, \delta_{S}, N_{S}\right)$ and $R_{2}\left(S, \delta_{S}, N_{S}\right)$ in Condition 1 and in turn $\delta_{S}$ will be updated to minimize $D_{0}$ further. So the two steps will be iterated to update $\delta_{S}$ and $N_{S}$ until $D_{0}$ has little change.

\section{B. Side Decoding Optimization With Prediction}

At the receiver, when only one channel works, the normal side decoder just uses the received description as the reconstruction. However, in our scheme, we know that far sublattice points received may produce much larger side distortions than the near ones. Therefore, it is not advisable to reconstruct the value $\lambda$ using the far sublattice point $\lambda_{2}^{\prime}$. In view that we only use 2-D vectors based on $A_{2}$ lattice, the correlation among wavelet coefficients is far from being exploited, especially those in the low frequency subbands. We may consider exploiting inter-vector correlation to improve the reconstruction quality by predicting the lost near sublattice points. Given our alternative transmission way, the lost near sublattice point for the received far sublattice point can be bi-directionally predicted from the two neighboring (the previous and the following) near sublattice points received in the same description. If the received far sublattice point can form a valid label (pair) with its predicted near sublattice point (the previous or the following), then a fine lattice point can be reconstructed as a forward or backward approximate value. However, if the label obtained by the combination of the predicted near sublattice point with the received far sublattice point is not valid, the forward or backward approximate value of $\lambda$ is the far sublattice point itself. The final reconstruction value from the side decoding is the average of forward and backward approximate values. It is noted that the prediction is performed for neighboring vectors in the same subband according to the directional correlation illustrated in Fig. 2. Another point to be noted is that the first or last sublattice point in a description has only backward or forward approximate value based on backward or forward prediction.

As an example, suppose channel 1 transmits the sequence of sublattice points starting with a near sublattice point and then followed by far and near sublattice points alternatively. Then channel 2 starts with a far sublattice point. As shown in Fig. 5, if only channel 1 works with the received sequence $\{\mathrm{O}$, A, O, B, O, B, D, B \}, based on our optimized side decoding with prediction, the reconstruction result is $\{\mathrm{O}, \mathrm{a}, \mathrm{O}, \mathrm{b}, \mathrm{O}$, midpoint of $b$ and $i, D, i\}$. Similarly if only $\{A, O, A, O$, $\mathrm{B}, \mathrm{O}, \mathrm{B}, \mathrm{D}\}$ is received from channel $2,\{\mathrm{a}, \mathrm{O}, \mathrm{a}, \mathrm{O}, \mathrm{b}, \mathrm{O}$, midpoint of $\mathrm{b}$ and $\mathrm{i}, \mathrm{D}\}$ is obtained as the reconstruction result. In fact, instead of directly using the near sublattice point as the reconstruction, one can also estimate the lost far sublattice point based on the same prediction rule, thus producing a likely better reconstruction by combining the received near sublattice point with the predicted far lattice point. However, a wrong prediction may lead to poorer reconstruction.

To substantiate the improvement of the proposed optimized side decoding over the normal side decoding, the following experiment was performed. Two standard images Lena $(512 \times 512)$ and Barbara $(512 \times 512)$ were directly encoded using MDLVQ with volume-size $\delta_{S}=5$ and various index numbers. Then they are decoded by the normal and the optimized side decoding respectively. Table II shows that with the same bit rate and same central PSNR values, the side PSNR values by the optimized side decoding scheme are much higher than using the normal side decoding. Moreover, the difference between side PSNR values from two channels with our proposed scheme is around $0.03 \mathrm{~dB}$, which shows a balance of reconstruction quality is also maintained.

With these encoding and decoding optimization schemes, the overall performance of our MDLVQ has improved substantially, especially the side distortion results as shown above. Inevitably computational complexity has also increased. The most timeconsuming part is the encoding optimization which involves the iterative processing for finding the two encoding parameters. The decoding optimization only requires some more table lookup operations for fine lattice point location and simple averaging calculations, the computational cost of which is negligible. In view that the similar encoding optimization for two encoding parameters is also employed in the optimized MDSQ scheme in [5], the computational complexity of the optimization schemes in our MDLVQ is almost the same as that of the optimized MDSQ scheme. 


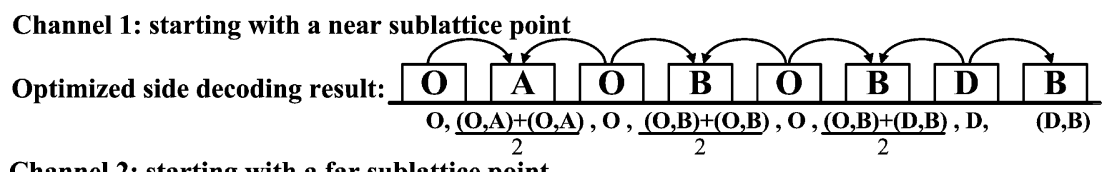

Channel 2: starting with a far sublattice point

Fig. 5. Side decoding with prediction for better reconstruction.

TABLE II

SidE PSNR VALUES OF NOMAL SCHEME VERSUS OPTIMIZED DECODING SCHEME

\begin{tabular}{|c|c|c|c|c|c|}
\hline \multicolumn{2}{|c|}{ Lena } & $\mathrm{N}=7$ & $\mathrm{~N}=13$ & $\mathrm{~N}=19$ & $\mathrm{~N}=31$ \\
\hline Normal & Side 1 & 35.621 & 31.357 & 28.686 & 25.521 \\
\hline$(\mathrm{dB})$ & Side 2 & 35.583 & 31.331 & 28.456 & 25.528 \\
\hline Optimized & Side 1 & 37.656 & 33.890 & 31.272 & 28.237 \\
\hline (dB) & Side 2 & 37.660 & 33.868 & 31.251 & 28.211 \\
\hline \multicolumn{2}{|c|}{ Barbara } & $\mathrm{N}=7$ & $\mathrm{~N}=13$ & $\mathrm{~N}=19$ & $\mathrm{~N}=31$ \\
\hline Normal & Side 1 & 35.797 & 31.248 & 28.393 & 24.844 \\
\hline$(\mathrm{dB})$ & Side 2 & 35.784 & 31.256 & 28.105 & 24.836 \\
\hline Optimized & Side 1 & 37.161 & 32.831 & 29.999 & 26.627 \\
\hline$(\mathrm{dB})$ & Side 2 & 37.175 & 32.846 & 29.998 & 26.643 \\
\hline
\end{tabular}

TABLE III

RESULTS OF OPTIMIZED PARAMETERS FOR "BARBARA"

\begin{tabular}{|c|c|c|c|}
\hline$\delta_{S}$ & $N_{S}$ & Central PSNR & Side PSNR \\
\hline 18.125 & $\begin{array}{c}7,7,7,7, \quad 7,7,7, \quad 7,7,7 \\
7,7,7\end{array}$ & $35.406 \mathrm{~dB}$ & $29.235 \mathrm{~dB}$ \\
\hline 17.8125 & $\begin{array}{c}7,7,7,7, \quad 7,7,7 \\
7,7,7, \quad 13,13,13\end{array}$ & $35.533 \mathrm{~dB}$ & $27.058 \mathrm{~dB}$ \\
\hline 17.1875 & $\begin{array}{l}7,7,7,7, \quad 7,7,7 \\
13,13,13,13,13,13\end{array}$ & $35.746 \mathrm{~dB}$ & $26 . .445 \mathrm{~dB}$ \\
\hline 16.25 & $\begin{array}{ll}7,7,7,7, & 7,7,7 \\
19,19,19, & 19,19,19\end{array}$ & $36.104 \mathrm{~dB}$ & $24.255 \mathrm{~dB}$ \\
\hline 15.9375 & $\begin{array}{ll}7,7,7,7, & 7,7,7 \\
31,31,31, & 31,31,31\end{array}$ & $36.241 \mathrm{~dB}$ & $21.694 \mathrm{~dB}$ \\
\hline
\end{tabular}

\section{EXPERIMENTAL RESULTS}

Two standard images Barbara $(512 \times 512)$ and Lena $(512 \times 512)$ are used to test our scheme against others. In this paper, we focus on the comparison of our proposed MDLVQ against the optimized MDSQ [5] since they are analogous except the quantization and related optimization schemes, although some other MD coding schemes are also included for reference. To make a fair comparison, the same experimental setup for the MDSQ scheme in [5] was applied here, i.e., 10/18 Daubechies wavelet with 4 levels was used for wavelet decomposition and target bit rate per channel is in the range $0.25-1$ bpp. In Table III, we present some example results of our optimized encoding parameters obtained for the standard images Barbara at the bit rate $0.5 \mathrm{bpp}$ per channel, where $N_{S}$

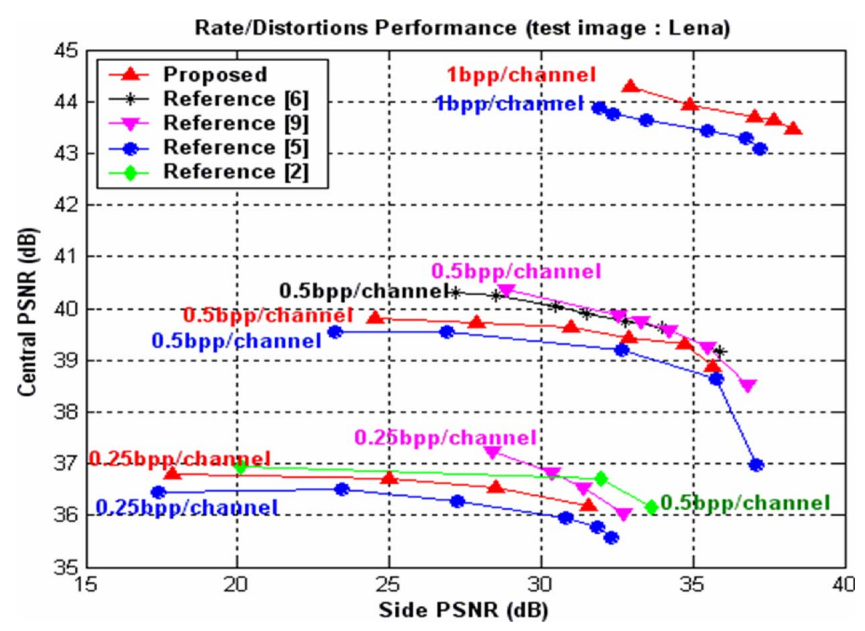

Fig. 6. Central and side PSNR results by the proposed coder and other referenced coders for "Lena."

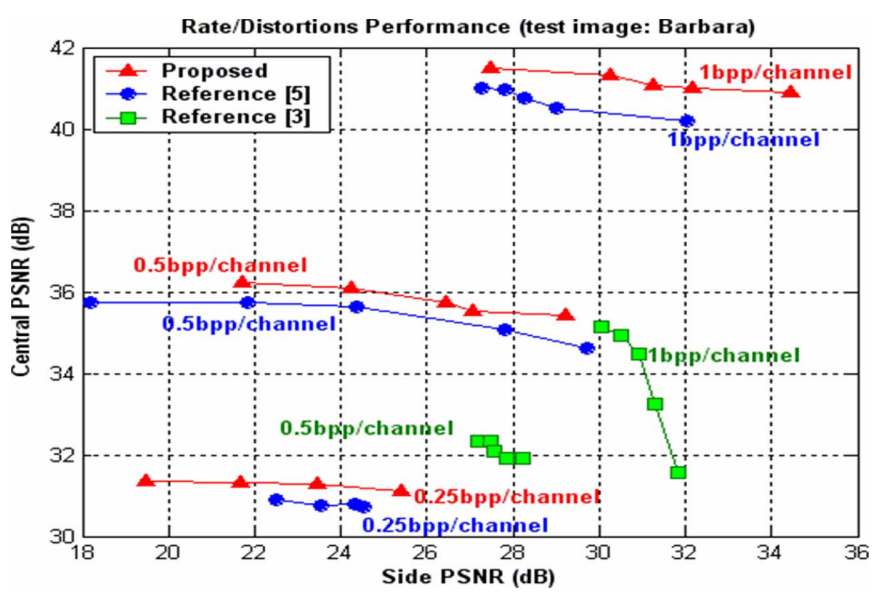

Fig. 7. Central and side PSNR results by the proposed coder and two referenced coders for "Barbara."

represents a set of the index numbers for 13 different subbands from low frequency subband to high frequency subbands, that is, LL4, HL4, LH4, HH4, HL3, LH3, HH3, . ., HL1, LH1, HH1.

From Table III, we can see at the bit rate 0.5 bpp per channel, while the volume-size $\delta_{S}$ decreases and the index number $N_{S}$ increases, the central PSNR values increase but the side PSNR values decrease, or vice versa. This is analogous to the variation of central and side PSNR values in MDSQ [5] as changing step size in SQ and the number of diagonals in MDSQ index assignment.

Figs. 6 and 7 show the central and side distortion performance of the proposed MDLVQ scheme against the optimized MDSQ [5] for the two tested images at the bit rates of $0.25,0.5$, and 
TABLE IV

RATE/Distortions PREFORMANCE FOR "BARBARA"

\begin{tabular}{ccccc}
\hline \hline Barbara & \multicolumn{2}{c}{ Proposed Scheme PSNR } & \multicolumn{2}{c}{ MDSQ [5] PSNR } \\
\hline Bit rate & Central & Side & Central & Side \\
$0.25 \mathrm{bpp}$ & $31.255 \mathrm{~dB}$ & $25.417 \mathrm{~dB}$ & $30.750 \mathrm{~dB}$ & $24.320 \mathrm{~dB}$ \\
$0.35 \mathrm{bpp}$ & $33.191 \mathrm{~dB}$ & $27.246 \mathrm{~dB}$ & $32.689 \mathrm{~dB}$ & $26.558 \mathrm{~dB}$ \\
$0.5 \mathrm{bpp}$ & $35.406 \mathrm{~dB}$ & $29.235 \mathrm{~dB}$ & $35.070 \mathrm{~dB}$ & $27.825 \mathrm{~dB}$ \\
$0.65 \mathrm{bpp}$ & $37.063 \mathrm{~dB}$ & $30.796 \mathrm{~dB}$ & $36.852 \mathrm{~dB}$ & $29.447 \mathrm{~dB}$ \\
$0.85 \mathrm{bpp}$ & $39.489 \mathrm{~dB}$ & $33.122 \mathrm{~dB}$ & $38.864 \mathrm{~dB}$ & $30.974 \mathrm{~dB}$ \\
$1 \mathrm{bpp}$ & $40.882 \mathrm{~dB}$ & $34.464 \mathrm{~dB}$ & $40.193 \mathrm{~dB}$ & $32.028 \mathrm{~dB}$ \\
\hline \hline
\end{tabular}

1 bpp per channel. Other two MD coders presented in [2] and [3] are also included for comparison. From the figures, we can clearly see that our proposed MDLVQ outperforms the tested MD image coders [2], [3]. Compared with the optimized MDSQ in [5], ours can still consistently improve around 0.2-0.4 dB for "Lena" and 0.5-0.7 dB for "Barbara" in central distortion with same or very close side distortions at the same bit rate. On the other hand, with similar central distortions, the proposed MDLVQ can achieve more improvement in side distortion compared with MDSQ [5], e.g., about 2-5 dB for "Lena" and 3-5 dB for "Barbara" in side distortion for some points in Figs. 6 and 7.

It can be seen from Fig. 6 that the two algorithms reported in [6] and [9] have shown better performance than our proposed MDLVQ scheme. The possible reasons may lie in a few aspects, among which a typical and crucial reason is that different single-description image coders and entropy coders are employed in the two MD coders. It is known that the JPEG-2000 standard is developed to achieve excellent rate-distortion performance for single-description image coding, especially at low bit rate. It is reasonably conjectured that the gain of the MD image coders in [9] based on JPEG-2000 may come largely from better performance in the coding of each individual description using JPEG-2000. On the other hand, the Tarp filter image coder with classification for embedding (TCE) coder [10] has also exhibited very good rate-distortion performance in single-description image coding, which is comparable to JPEG-2000. The MD image coder in [6] is developed based on a modified MDSQ (MMDSQ) together with the TCE entropy coder, denoted as MMDSQ-TCE. It is explicitly stated in [6] that the efficiency of MMDSQ-TCE is partially due to the efficiency of the TCE coder, while the modified MDSQ adapts the efficient image coder naturally to an MD system.

To have a closer look at the different performance between the proposed MDLVQ scheme and the optimized MDSQ, Table IV tabulates some representative pairs of central and side distortion results with respect to various bit rates for the image "Barbara." These representative pairs are points with good balance between central and side distortions in both schemes. From the results, we can find that our proposed MDLVQ consistently outperforms the optimized MDSQ [5] in both central and side distortion si- multaneously at the same bit rate over a wide range from 0.25 to 1 bpp per channel, with both improvements of $0.688-2.436 \mathrm{~dB}$ in side distortion and $0.211-0.689 \mathrm{~dB}$ in central distortion.

\section{CONCLUSION}

An MD image coding scheme using MDLVQ has been developed in the paper. Effective optimization schemes in both MDLVQ encoding and decoding have been accommodated in the proposed system to achieve better rate and central/side distortions performance. From the appropriate vector construction to the optimization of encoding parameters and optimized side decoding, the proposed MDLVQ has demonstrated superior rate-distortion performance to some other tested MD image coders including the optimized MDSQ image coder in [5]. Furthermore, in view of the fast LVQ encoding and similar computational complexity to the optimized MDSQ image coder in [5], our MDLVQ scheme is a worthy choice for MD coding.

\section{ACKNOWLEDGMENT}

The authors would like to thank the anonymous reviewers for their valuable comments that greatly improved this paper.

\section{REFERENCES}

[1] V. K. Goyal, "Multiple description coding: Compression meets the network," IEEE Signal Process. Mag., vol. 18, no. 5, pp. 74-93, Sep. 2001.

[2] Y. Wang, M. T. Orchard, and A. R. Reibman, "Multiple description image coding for noisy channels by pairing transform coefficients," in Proc. 1st Workshop Multimedia Signal Process., Princeton, NJ, Jun. 1997, pp. 419-424

[3] M. Srinivasan and R. Chellappa, "Multiple description subband coding," in Proc. IEEE Int. Conf. Image Processing, Chicago, Oct. 1998, vol. 1, pp. 684-688.

[4] S. D. Servetto, V. A. Vaishampayan, and N. J. A. Sloane, "Multiple description lattice vector quantization," in Proc. IEEE Data Compression Conf., Snowbird, UT, Mar. 1999, pp. 13-22.

[5] S. D. Servetto, K. Ramchandran, V. A. Vaishampayan, and K. Nahrstedt, "Multiple description wavelet based image coding," IEEE Trans. Image Process., vol. 9, no. 5, pp. 813-826, May 2000.

[6] C. Tian and S. S. Hemami, "A new class of multiple description scalar quantizer and its application to image coding," IEEE Signal Process. Lett., vol. 12, pp. 329-332, Apr. 2005.

[7] V. A. Vaishampayan, N. J. A. Sloane, and S. D. Servetto, "Multiple description vector quantization with lattice codebooks: Design and analysis," IEEE Trans. Inf. Theory, vol. 47, pp. 1718-1734, Jul. 2001.

[8] H. Bai, Y. Zhao, and C. Zhu, "Optimized multiple description image coding using lattice vector quantization," in IEEE Int. Symp. Circuits Syst., Kobe, Japan, May 2005, vol. 4, pp. 4038-4041.

[9] T. Tillo and G. Olmo, "A novel multiple description coding scheme compatible with the JPEG2000 decoder," IEEE Signal Process. Lett., vol. 11, pp. 908-911, Nov. 2004.

[10] C. Tian and S. S. Hemami, "An embedded image coding system based on Tarp filter with classification," in Proc. Int. Conf. Acoustics, Speech, Signal Process., Montreal, QC, Canada, May 2004, vol. 3, pp. 49-52.

[11] J. M. Shapiro, "Embedded image coding using zerotrees of wavelet coefficients,” IEEE Trans. Signal Process., vol. 41, pp. 3445-3462, Dec. 1993.

[12] J. H. Conway and N. J. A. Sloane, Sphere Packings, Lattices and Groups, 3rd ed. New York: Springer-Verlag, 1998, pp. 108-117.

[13] M. Nelson and J. Gailly, The Data Compression Book, 2nd ed. New York: M\&T Books, 1995, ch. 4. 